21.2

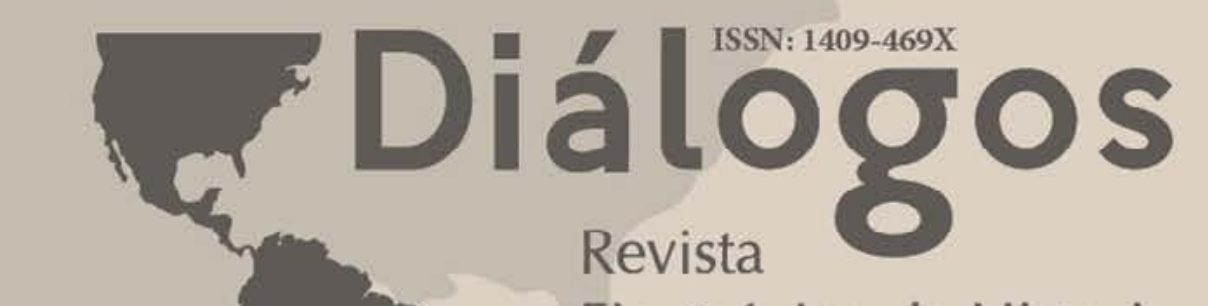

Electrónica de Historia

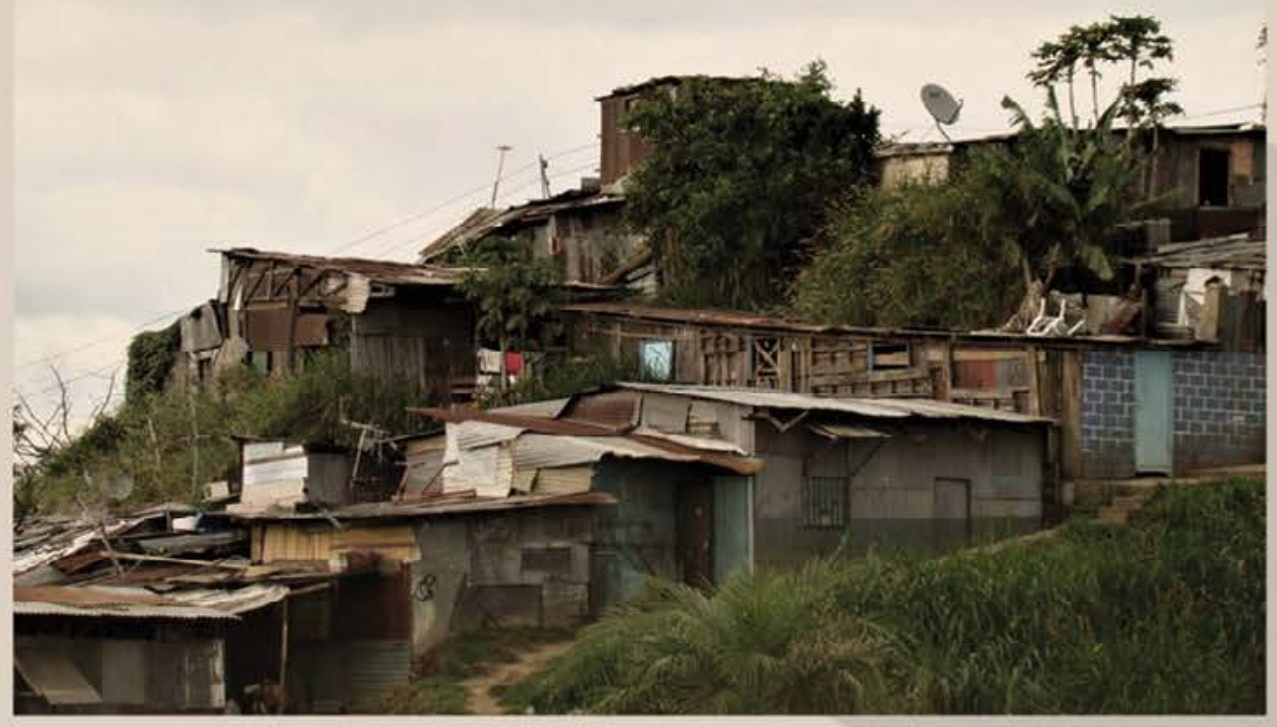

Centro de Investigaciones Históricas de América Central. Universidad de Costa Rica Julio-diciembre 2020

url: http://revistas.ucr.ac.cr/index.php/dialogos/index
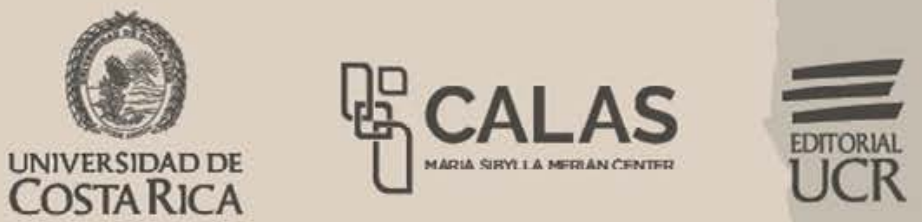


\title{
MULTIFUNCIONALIDAD DE LA VIDA CAMPESINA EN CONTEXTOS DE CONFLICTOS AMBIENTALES Y CONSTRUCCIÓN DE PAZ EN LA AMAZONÍA COLOMBIANA.
}

\author{
Sara Camila Arias
}

\begin{abstract}
Resumen
Las dinámicas extractivas en la Amazonía colombiana fueron frenadas parcialmente por la dinámica de guerra presente en el país. Con el cese al fuego, aunado a la insuficiente presencia estatal y las persistentes condiciones de desigualdad social y también la continuidad de otros actores armados, esta área es escenario de economías extractivas e ilegales al tiempo que permanece siendo fuente de supervivencia para las comunidades campesinas que han habitado esas tierras durante décadas y se han aproximado a una forma de relacionamiento equilibrada con el ecosistema. Ante este contexto de conflictividad, el Estado ha decidido criminalizar cualquier tipo de intervención, incluyendo la de las comunidades locales históricamente asentadas allí, y también ampliar las áreas protegidas, lo cual se ha acompañado de la militarización de su protección que se conecta con la judicialización a quienes sean considerados actores de deforestación. A pesar de esta nueva dinámica de conflicto, ya no armado sino social y ambiental, las comunidades campesinas y las personas en proceso de reincorporación le apuestan a la construcción de paz, defendiendo su territorialidad e impulsando diferentes proyectos productivos que hacen sinergia en el contexto de la multifuncionalidad de la vida campesina de la que ahora forman parte.
\end{abstract}

Palabras clave: conservación ambiental, consolidación de paz, Amazonia,clase campesina.

Fecha de recepción: 26 de diciembre de 2019 • Fecha de aceptación: 20 de mayo de 2020

Sara Camila Arias - Colombia. Universidad Nacional de Colombia. Politóloga, Magister en Medio Ambiente. Con experiencia en trabajo comunitario con víctimas del conflicto armado, comunidades campesinas y con población de FARC en proceso de reincorporación, orientado a iniciativas para su vida digna y fortalecimiento organizativo. Contacto: scariasc@unal.edu.co

ORCID: https://orcid.org/0000-0002-0689-0169 


\title{
MULTIFUNCTIONALITY OF RURAL LIFE \\ WITHIN THE CONTEXTS OF ENVIRONMENTAL CONFLICTS AND PEACEBUILDING IN THE COLOMBIAN AMAZON
}

\begin{abstract}
The extractive dynamics in the Colombian Amazon were partially halted by the dynamics of war present in the country. With the ceasefire, the insufficient state presence, the persistent conditions of social inequality and the continuity of other armed actors, this area is scene of extractive and illegal economies while remaining a source of survival for the peasants communities that have inhabited these lands for decades and have approached a balanced relationship with the ecosystem. Given this context of conflict, the State has decided to criminalize any type of intervention, including activities of the local communities historically settled there. It also has expanded the protected areas, which has been accompanied by the militarization of its protection and relates to the judicialization of those who are considered deforestation actors. Despite this new conflict dynamic, which is no longer armed but social and environmental, peasant communities and people in the process of reincorporation bet on peacebuilding, defending their territoriality and promoting different productive projects that make synergy in the context of the multifunctionality of the peasant life in which they are now part.
\end{abstract}

Keywords: environmental conservation, peacebuilding, Amazonia, peasant class. 


\section{INTRODUCCIÓN}

El $81 \%$ de los conflictos armados que se dieron en el mundo entre 1950 y 2000 se dieron en 23 de las 34 zonas de alta biodiversidad identificadas por Hanson et al. (2009), entre las que se encuentran los andes tropicales. En Colombia, esta realidad se presenta con la paradoja de que, si bien los grupos armados ilegales se sirvieron del gravamen o explotación de actividades extractivas en estas áreas, también su presencia evitó la incursión a gran escala de economías de este tipo. En detalle, existe una relación multidimensional entre el medio ambiente y el conflicto armado. Esta premisa se puede desagregar en los siguientes factores explicativos, no sin antes aclarar que por medio ambiente se entienden las relaciones ecosistema-cultura, comprendiendo a ésta última como plataforma adaptativa (Maya, 2013):

i) el conflicto armado se desenvolvió fundamentalmente sobre escenarios de alta biodiversidad cubiertos por bosques o selvas tropicales,

ii) el control y la explotación de los recursos naturales están intrínsecamente relacionados con la guerra, la disputa por ellos ha jugado un papel importante en el surgimiento y mantenimiento del conflicto, o son el medio a través del cual los actores del conflicto obtienen el financiamiento necesario para sostenerlo,

iii) la voladura de oleoductos, la deforestación asociada a los cultivos de uso ilícito y la minería ilegal son algunos de los efectos que han dejado estas décadas de conflicto sobre sobre los ecosistemas, la biodiversidad, los recursos naturales que hace del medio ambiente una víctima del conflicto, y finalmente,

iv) los actores armados, paradójicamente, han impuesto barreras de hecho a la entrada de economías extractivas a gran escala sobre áreas altamente biodiversas y, en cierta medida, han tenido un efecto ambiental preservador y conservado.

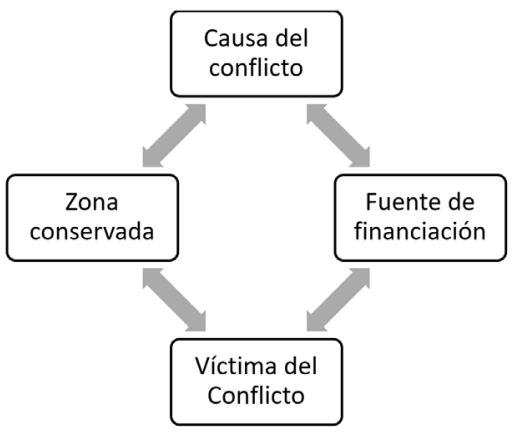

Figura 1. Relaciones Ecosistema-Cultura en escenarios de conflicto armado. Fuente: Elaboración propia.

Lo anterior significa que, si bien en algunos casos la relación se limita a la disputa por la extracción de recursos naturales como causa del conflicto armado, el conjunto de relaciones expuestas aborda aspectos mucho más complejos de la relación entre ambiente y conflicto armado (Rodríguez, Rodríguez \& Durán, 2017). 
Esta complejidad también será un factor en los procesos de construcción de paz en las zonas con alta biodiversidad, pues las acciones de las comunidades campesinas que allí se despliegan no se centraron en sobrevivir, sino que trascendieron a un plano de exigibilidad que representó la voluntad de generar cambios, procesos sociales y dignificar la vida. Esta característica hace que el ecosistema pase a configurarse como lugar de resolución de necesidades, de consecución de derechos y, en general, de bienestar y buen vivir. Por ello, se identifica que la implementación real y efectiva del Acuerdo de Paz aportaría a la consolidación y fortalecimiento de estas iniciativas sociales, lo cual sin duda transforma el ecosistema con cambios apalancados por la inversión de recursos institucionales que se derivan del mismo proceso de implementación del Acuerdo.

En particular, la amazonia colombiana se ubica en la periferia de la geografía nacional, vivió el conflicto armado con mayor intensidad y coincide con una de las zonas más biodiversas, la cual recientemente ha sido relevante para las acciones estatales de conservación ambiental que responden al cumplimiento de los compromisos internacionales asumidos por el país. Estos se han concentrado en la ampliación y creación de áreas protegidas (AP) y en una política de judicialización de los actores que son considerados responsables de la degradación de éstas, para la cual se sirven de recursos militares para llegar a los territorios.

Para comprender de manera consistente esta situación, a continuación se expone cómo se entiende la amazonia colombiana como un territorio de frontera para luego explicar su correspondencia con la visión de conservación que tiene el Estado. Lo anterior en contraposición con la visión integradora que asumen las comunidades campesinas que han habitado históricamente esta zona, la cual se comprende desde la multifuncionalidad de su proceso productivo que se ha dado en relación con el conflicto armado interno y ahora con la construcción de paz que permitió el cese al fuego y el proceso de reincorporación que allí se desarrolla.

\section{TERRITORIO DE FRONTERA Y ECONOMÍAS DE ENCLAVE.}

Colombia se ha caracterizado por altos índices de concentración de la propiedad rural que se remontan a los siglos XVII y XIX durante la colonia. Esta característica no cambió en lo que se denominó la república y hoy se configura como una de las causas del conflicto social y armado. Lo anterior condujo a modelos de ocupación territorial por parte de campesinos en busca de espacios para poder cultivar y continuar con su manera de ser y vivir, con y en el ambiente, que a su vez condujo a la expansión de la frontera agrícola.

La expansión de la frontera agrícola no se explica de manera monocausal como la configuración de lugares de extensión del Estado central, vacantes y a la espera de su ocupación y administración. Por el contrario, se trata de territorios en los que el conflicto social se asienta y reproduce: 
Datos recientes confirmaron la ocurrencia en áreas colonizadas de las mismas tendencias observadas en las agrícolas: la descomposición del campesinado y la concentración de las tierras. El colono campesino no migra solo. La migración tampoco tiene lugar en un vacío social. Más bien, los colonos que se mudan a nuevas áreas reproducen las relaciones sociales campesinas de las producciones en la frontera. En efecto, el colono no crea un nuevo espacio libre en la frontera; simplemente amplía el contexto geográfico en el que los campesinos son dominados (Domínguez citado en Uribe, 2013).

De esta manera, los territorios de colonización campesina han sido espacios que siguen la lógica de enclave y del desarrollo capitalista en términos amplios: allí se impone un orden -sobre un territorio considerado caótico- para el uso racional (explotación minera, agroindustrial o relacionadas / conservación-green grabbing) de un recurso natural (Serje, 2005). En dicho orden, el componente humano es secundario, lo que se traduce en desplazamientos masivos de población o amenazas a la seguridad de los habitantes, desde todas las dimensiones, quienes terminan siendo perseguidos por garantizar sus condiciones de vida.

Con estas territorialidades construidas se confirma la persistencia del mito de la frontera en el que estos territorios vastos, donde predominaba el refugio y la resistencia, se "configuraron áreas escasamente pobladas, cuyos habitantes estaban dispersos o eran itinerantes en un territorio muy grande, de fronteras abiertas y de difícil comunicación" (Serje, 2005, p. 156). De manera relacionada, algunos historiadores han considerado los aspectos geográficos como determinantes en la construcción de la nación colombiana, tanto así que se consideró que este elemento condicionó de cierta manera las características sociales y culturales de la sociedad colombiana y de la relación del estado centralista con territorios de frontera (Safford \& Palacios, 2012). Según Serje (2005), los territorios de frontera

\footnotetext{
han sido precariamente apropiados por los pobladores ancestrales; poco modificados por la labor humana y definitivamente desintegrados del sistema económico nacional. Lo que afianza la idea de tierras disponibles, habitadas por una población de cierta manera superflua, cuya ocupación puede definitivamente ser ignorada o desechada. (p. 156)
}

Es innegable que el poblamiento de estas zonas tiene mucho que ver con una estructura agraria que ha privilegiado al latifundio y con una dinámica de violencia sistemática contra los pobladores rurales empobrecidos. La violencia creciente en los campos de muchas zonas del país provocó el desplazamiento y la búsqueda de nuevos lugares de vida, asumiendo áreas apartadas de frontera, como refugio de su vida y su cultura, las cuales luego pasaron a ser declaradas protegidas.

Es así como muchas de estas nuevas ocupaciones se dieron en ecosistemas estratégicos que luego fueron definidos como áreas protegidas en las que desde la normatividad colombiana se prohíben los usos productivos y la habitabilidad de estas. Esto permite proponer que la definición de las zonas destinadas a la conservación se pudo dar sin la participación de las comunidades que allí habitaban y que predominó 
una visión en la que cualquier intervención humana implica una degradación a la base natural. Por lo cual, se concluye que hay un escenario de conflictividad marcado por el desconocimiento de la relación ecosistema-cultura de las múltiples formas de producción y a la vez de como mantener un equilibrio en la composición, funcionamiento y estructura de los ecosistemas, y de los derechos de las comunidades establecidas en estos territorios.

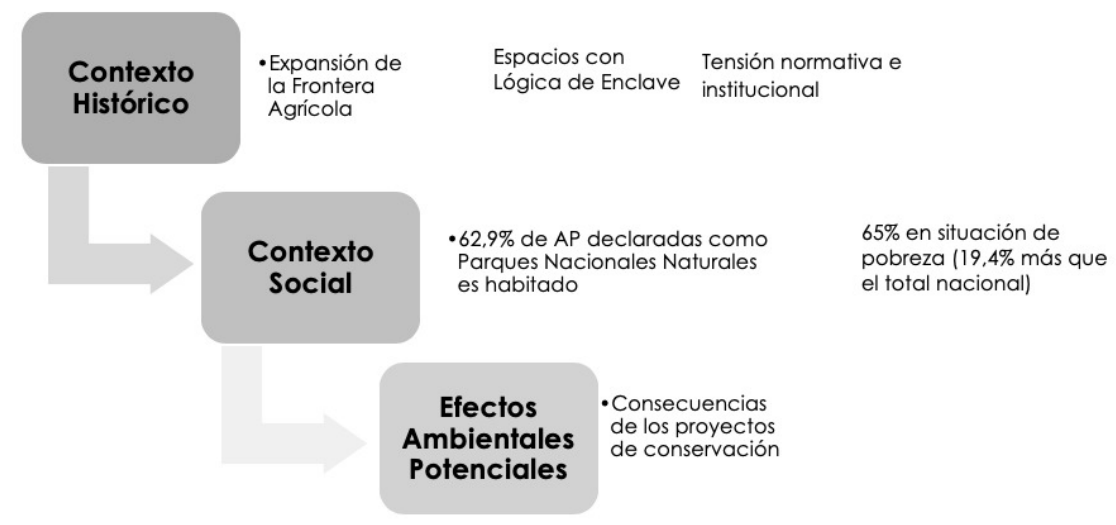

Figura 2. Escenario de conflictividad en territorios de frontera declarados AP. Fuente: Elaboración propia.

Es así como las áreas protegidas en Colombia se diseñaron como lugares para el aislamiento de los ecosistemas de la sociedad, visión que desconoce que la conservación de la biodiversidad en dichas áreas ocurrió con la participación de las cosmogonías, conocimientos y saberes tradicionales de las comunidades asentadas en estas áreas. Por esta razón, se hace imperativo avanzar hacia nuevas formas de ordenamiento ambiental y social de las áreas protegidas, en las que se reconozca que los habitantes pueden ser sujetos activos en la conservación y pueden garantizar una zonificación ambiental en la que no existe una restricción para la integración de las funciones ecológicas y sociales de estas áreas.

Entonces, en los territorios de frontera gran parte de las políticas de desarrollo económico están en función de nuevas dinámicas extractivas entre las que se distinguen el acaparamiento de tierras, minería, agroindustria a gran escala y proyectos de conservación, las cuales mantienen una visión de territorio por ordenar y una población por controlar sea para la implementación de modelos de agroindustria o de visiones de conservación de una naturaleza prístina. 


\title{
¿CONSERVACIÓN PARA QUÉ?
}

El Estado, a través de dispositivos legales y de política argumentados desde el paradigma tradicional de conservación de islas de naturaleza prístina, rompe con las relaciones ecosistema-cultura y niega la posibilidad de una continuidad en el relacionamiento entre la sociedad y la naturaleza aun cuando el componente cultural es parte de las relaciones, funciones y estructura del ecosistema (Posey, 1985). Lo anterior al definir que en ciertas áreas se prohíbe la vida humana que ya se venía desarrollando, proponiendo como alternativa su reasentamiento. Es decir, rompe la matriz que concibe ecología y cultura como integrantes e interdependientes del ambiente. Como consecuencia asume que la forma válida de conservar la biodiversidad es separando a las comunidades del ecosistema. A través de estos dispositivos, se aísla al ser humano de la naturaleza y a la cultura del ecosistema. La conservación desde este paradigma

\begin{abstract}
se asimila a un culto de la vida silvestre, el cual es implantado para promulgar la defensa de la naturaleza prístina y justificar la preservación inmaculada del mundo natural, la valoración científica, la admiración estética del paisaje, e incluso, la expectativa de usos futuros de los recursos (Martínez, 2004).
\end{abstract}

Aunado a esto, existe una debilidad institucional del Estado que ha ocasionado desconfianzas mutuas en las que ninguna de las partes es beneficiada: las áreas no se conservan y la comunidad es estigmatizada y criminalizada. Por lo tanto, crear sinergias entre estos actores, reconocerles sus derechos y hacerlos aliados en la conservación de una manera integral y efectiva, es decir permitiéndoles desarrollar su vida allí, contribuye a conciliar los intereses en el territorio y a solventar la situación de conflictividad en estas áreas.

Ahora bien, ser conscientes de que la mayoría de las Áreas destinadas a protección o conservación están habitadas hizo que el paradigma tradicional reconociera esta presencia, normativamente sólo en los casos de indígenas y negros. Sin embargo, este cambio, lejos de transformar los dispositivos de control biológico ya mencionados, puede extenderlos hacia las poblaciones humanas: "el conocimiento detallado de la población cuyas vidas se ven afectadas por el establecimiento y ordenación de los parques es una información tan importante como la que se refiere a las plantas y animales que se han de conservar" (McNeely, 1994. s.p).

Es así como este "viraje" en el paradigma convierte a la población en un dato y un instrumento importante para la conservación, sea como objetos de estudio al asumirlos como informantes etnobotánicos o como objetos de trabajo al considerarlos como guardabosques (Beltrán, 2016, p. 221). Entonces, las comunidades pasan a ser los ejecutores de los proyectos diseñados y dirigidos por los especialistas y controlados por una institución estatal. Por esta razón, un verdadero cambio en el paradigma de la conservación debe reconocer integralmente los conocimientos y saberes tradicionales de las comunidades y, sobre todo, comprender y respetar la complejidad de sus relaciones con la naturaleza que han permitido permanezca en su composición, función y estructura. 


\begin{tabular}{|l|l|l|}
\hline Eje de Discusión & Paradigma Tradicional & Paradigma Integrador \\
\hline $\begin{array}{l}\text { Objeto de } \\
\text { conservación }\end{array}$ & Biodiversidad & $\begin{array}{l}\text { Diversidad biológica, } \\
\text { cultural y agraria }\end{array}$ \\
\hline $\begin{array}{l}\text { Objetivo de la } \\
\text { conservación }\end{array}$ & Para los seres humanos & Con los seres humanos \\
\hline Sostenibilidad & Débil & Fuerte \\
\hline $\begin{array}{l}\text { Población } \\
\text { Humana }\end{array}$ & $\begin{array}{l}\text { Agente devastador que } \\
\text { debe ser expulsado }\end{array}$ & $\begin{array}{l}\text { Agente de conservación } \\
\text { que debe ser integrado }\end{array}$ \\
\hline $\begin{array}{l}\text { Relación } \\
\text { ecosistema cultura }\end{array}$ & Escindida & Integradora \\
\hline Derecho a tutelar & Ambiente sano & $\begin{array}{l}\text { Derechos humanos y al } \\
\text { territorio }\end{array}$ \\
\hline $\begin{array}{l}\text { Producción del } \\
\text { conocimicnto }\end{array}$ & $\begin{array}{l}\text { Lineal unidireccional en } \\
\text { la relación sujeto-objeto }\end{array}$ & $\begin{array}{l}\text { Reconocimiento y } \\
\text { diálogo horizontal de } \\
\text { conocimientos }\end{array}$ \\
\hline
\end{tabular}

Figura 3. Síntesis de ejes de discusión entre paradigmas de conservación. Fuente: Elaboración propia.

De la dinámica que ha ocasionado esta visión de conservación se puede concluir que:

i) Las restricciones de usos entran en contradicción con las prácticas tradicionales de producción y estrategias de sobrevivencia al solo permitir actividades de conservación, recuperación, control, investigación, educación, recreación y cultura, olvidando los procesos históricos de territorialización.

ii) Las restricciones de adjudicación de baldíos al interior de las áreas protegidas convierten a los ocupantes de predios baldíos, que eran adjudicables en indebidos ocupantes de predios, ahora inadjudicables y hace que pierdan la posibilidad de adquirir la propiedad de la tierra independientemente del tiempo que lleven de ocupación.

iii) Las normas de las áreas protegidas entran en contradicción con derechos fundamentales de las comunidades, quienes se reconocen como parte integrante del territorio con un arraigo expresado en su relacionamiento con el ecosistema (que este paradigma de conservación restringe).

iv) La autoridad ambiental enfrenta la contradicción de tener que aplicar la normativa prohibitiva para la protección del área y, al mismo tiempo, dar viabilidad a la política de participación social en la conservación como estrategia de manejo de las áreas.

Reconocer esta situación condujo a replanteamientos en la visión de la conservación, incluyendo a la diversidad cultural y agrícola como objetos de conservación que contribuyen al mantenimiento de los ecosistemas y su biodiversidad. Las islas de naturaleza "prístina" no son impermeables o inmunes a los procesos de deterioro que tienen lugar en los ámbitos externos, sino que son sistemas abiertos inmersos en una cierta escala de espacio planetario (Toledo, 2005, p. 74), que integran y son integrados por construcciones socioculturales. 


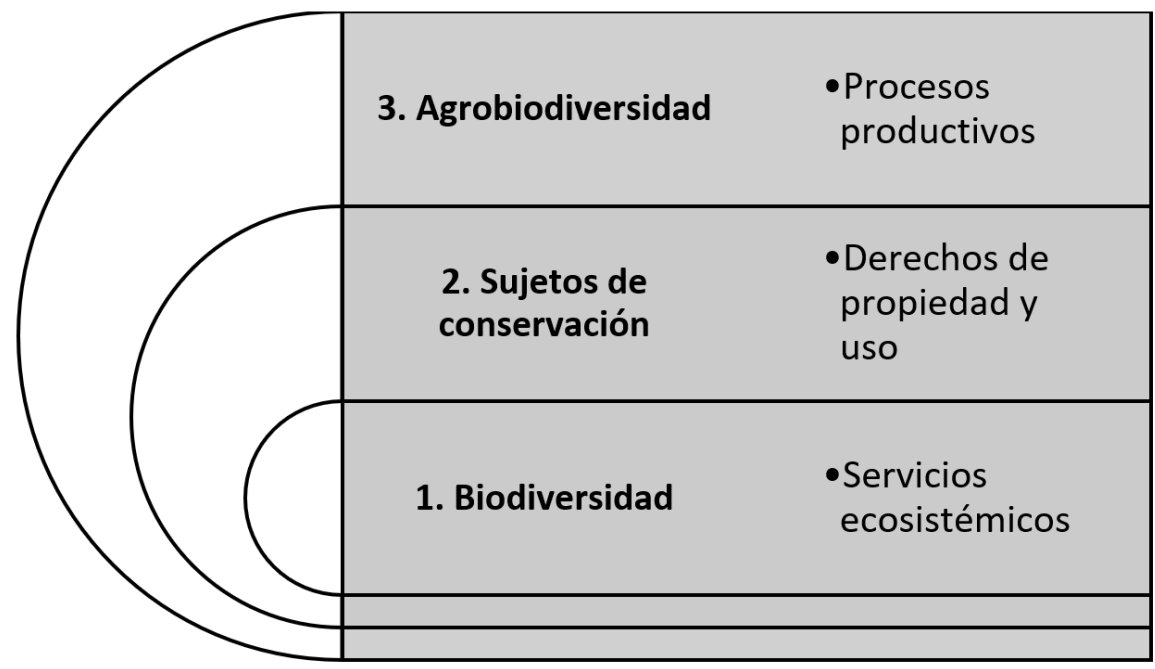

Figura 4. Ampliación de los objetos de la conservación. Fuente: Elaboración propia

Es un llamado a recordar que la tierra es un sistema ecológico dinámico compuesto también por una comunidad moral de la que todos formamos parte (Leopold, 2003). Por lo tanto, la conservación de la biodiversidad empieza a tomar en cuenta el conjunto de factores sociales que la condicionan (Toledo, 2005, p. 70). Incluso, desde el Convenio de las Naciones Unidas sobre la Diversidad Biológica (CDB), se menciona que las comunidades locales que habitan zonas protegidas juegan un rol fundamental en la conservación del medio ambiente y no deben ser vistas como actores perjudiciales para la consecución de los objetivos de conservación de las AP. Por el contrario, estos son agentes que hacen parte del ecosistema y que deben ser tenidas en cuenta como sujetos activos en los planes de manejo de estas áreas.

Para reforzar esta idea, es pertinente mencionar algunos estudios (Denevan, 1992; Gómez-Pompa \& Kaus, 1992; Berkes \& Davidson-Hunt, 2006; Posey, 1985,1997 citados por Nemogá, 2016) que demuestran que los pueblos nativos coevolucionaron con la selva húmeda y que fueron fundamentales para la proliferación de especies de semillas. Ello argumenta que la especie humana no es separada de la naturaleza y que sus prácticas productivas condujeron a la conservación de la biodiversidad.

Estos estudios pueden interpretarse bajo el enfoque biocultural, años más tarde descrito por Maffi (2010), quien lo define como la comprensión de la interrelación de la vida en sus manifestaciones biológicas, culturales y lingüísticas, las cuales han coevolucionado dentro de complejos sistemas adaptativos socio-ecológicos. Comprender estas interrelaciones avanza en el encontrar formas apropiadas de vivir con la naturaleza y argumenta la relevancia del valor intrínseco de la biodiversidad. 
Desde esta visión, la conservación puede ser más eficaz, ética y justa si las acciones se enfocan a contrarrestar simultáneamente la erosión biológica y cultural (Turner, Boelscher \& Ignace, 2000; McShane et al., 2011; Davidson-Hunt et al., 2012; Nemogá, 2016), teniendo en cuenta que hay estudios que demuestran un correlato entre la entre la pérdida de la diversidad cultural o lingüística y de la diversidad biológica en nuestro planeta (Persic \& Martin, 2008; Oviedo, Maffi \& Larsen, 2000). Existe otra diversidad que ha sido también menguada y degradada: la agrícola. Al respecto vale decir que ésta es el resultado de la selección artificial a la que los seres humanos han sometido a diferentes especies animales y vegetales con el fin de mejorar el acceso a alimentos, materias primas y fuerza para realizar trabajos: "la agrobiodiversidad es biodiversidad elaborada por el hombre" (Boyce, 2013).

La homogenización de los procesos productivos de la agricultura moderna amenaza esta agrobiodiversidad, la cual no tiene la capacidad de recuperarse por sí sola (Wolff, 2004). No basta con que se frene la expansión y la presión sobre esta con la agricultura tradicional, también es necesario valorar, rescatar y cuidar las prácticas culturales que están directamente relacionadas con ella, puesto que es la economía campesina la que abastece alimentariamente al planeta y la que a su vez es la más productiva; por ejemplo, una pequeña parcela produce más de lo que resulta en grandes extensiones de monocultivos (Grain, 2014).

Entonces, las causas de la pérdida de biodiversidad son también las causas de la pérdida de diversidad cultural y agroalimentaria. Esta afirmación conduce a pensar que las medidas deben ser coherentes y articuladas, reconociendo la importancia que reviste la protección de la biodiversidad para la "agricultura, la seguridad alimentaria y los medios de subsistencia en las zonas rurales, sobre todo de las poblaciones que viven en entornos marginales y adversos" (FAO, 2004).

Bajo esta mirada integradora cabe señalar alternativas, como la de restauración ecológica, que consideran a la intervención humana como capaz de proteger la biodiversidad, lo cual se acompasa con la comprensión del proceso de territorialización que emprenden las comunidades en su relación con el ecosistema y aclara que no solo hay despojo al negar el derecho de propiedad sino también cuando se da un cambio en el uso.

El campo de la restauración ecológica puede ser asumida como una estrategia de conservación que actúa como bodega de materias primas a futuro en la que unos especialistas diseñan un plan. Este plan puede que tenga en cuenta los conocimientos de las comunidades, incluso que las involucre en este proceso, o puede ser asumida como una práctica que aporte a la autonomía ambiental de las comunidades, que responda a sus necesidades y contexto local y que sea dirigida por ellas, en las que su visión de territorio y su compromiso por su sustentabilidad es también la garantía de su supervivencia. 


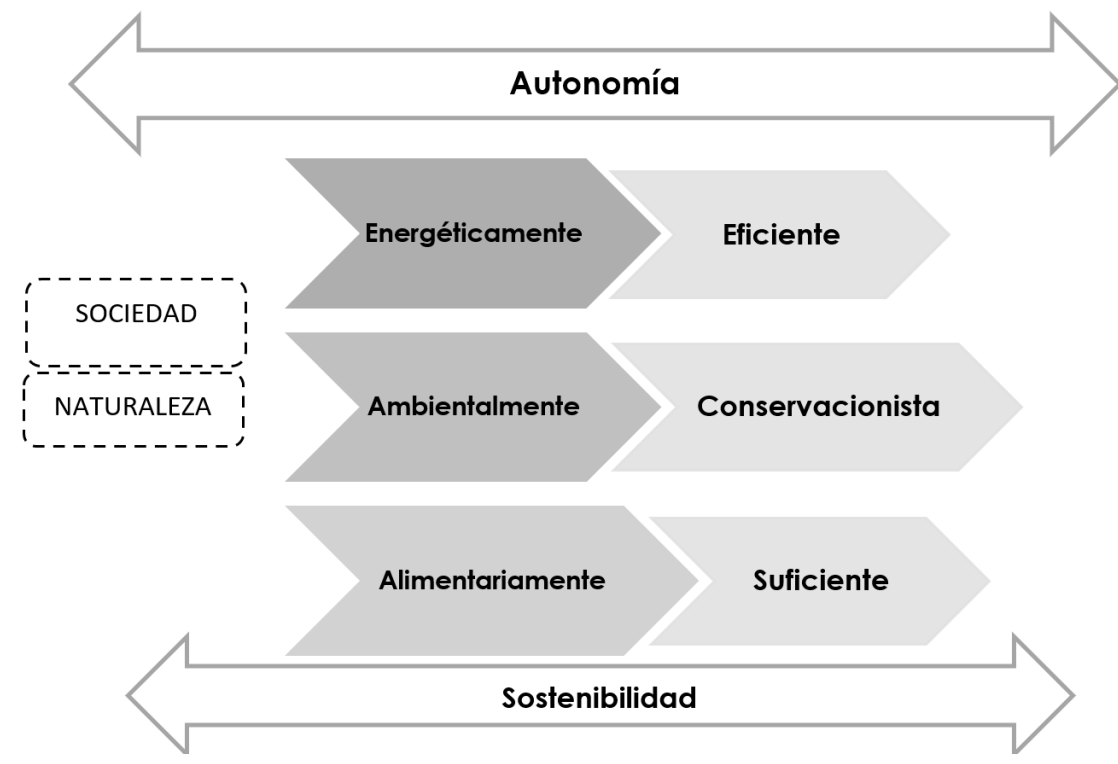

Figura 5. Integralidad de los procesos de Restauración Ecológica. Fuente: Elaboración propia.

En América Latina, la restauración ecológica se encuentra atravesada por diferentes relaciones y dinámicas de poder que dan como resultado que se configure como una muestra más de las disputas que se tejen en el continente por la definición de los usos de la tierra y de la apropiación o no de la biodiversidad. Entonces, una práctica de la restauración que reconozca las interacciones simultáneas y constitutivas entre ecosistema y cultura podrá asegurar desde la óptica de la justicia ecológica la garantía de la vida, humana y no humana (Gudynas, 2011).

Con esta práctica se abre la oportunidad de reconocer y validar públicamente culturas tradicionales en su potencialidad de generar y sostener relaciones equilibradas y en armonía con los ecosistemas, lo cual pasa por consolidar a las economías solidarias soportadas en una producción consciente de sus procesos de apropiación, transformación y excreción, en donde sus productos se articulan a mercados locales y regionales. Es así como la restauración ecológica se concibe como un proceso integral e integrador en el que se tienen en cuenta las necesidades humanas, individuales y colectivas, y la sostenibilidad de los ecosistemas. Siendo, en este sentido, un catalizador de sus procesos por la autonomía ambiental, económica y alimentaria. Esto implica que se avance en una nueva matriz de comprensión del problema ambiental, en el que las prácticas de restauración ecológica respondan no sólo a un compromiso por la participación de las comunidades y el reconocimiento de sus saberes, sino que se cuestionen los fundamentos epistemológicos sobre los que se sustentan, es decir, que avancen por un camino transdisciplinar en el que se reconozcan las prácticas ambientales comunitarias como parte de su organización social y de su acción política (Ploeg, 2010; Santacoloma, 2015). 


\section{MULTIFUNCIONALIDAD DE LA VIDA CAMPESINA Y CONSTRUCCIÓN DE PAZ}

A partir de la visión integradora de la conservación, se da paso a comprender una racionalidad diferente de la acción productiva, lo cual permite disgregar lo que se denomina una relación multidimensional con el territorio. Las comunidades campesinas están obligadas, por sus condiciones de marginalidad y abandono estatal, a adoptar formas de producción/apropiación que maximicen la unidad de producción y de recursos disponibles. Esta forma de ser, estar y adaptar en el ecosistema demuestra un alto grado de autosuficiencia en el que se mantienen la heterogeneidad espacial y diversidad biológica. Esta estrategia multiuso permite gestionar unidades geográficas con sus componente físicos y bióticos. Es así como los campesinos se resisten a la especialización de sus espacios naturales y de sus actividades productivas, pues esto los ubica en un mayor nivel de riesgo para su subsistencia (Toledo, 1993).

No se trata de que la economía campesina sea per se sostenible o no, sino de que las prácticas que se presentan en la unidad de producción aporten al equilibrio de los intercambios de materiales y energía y, en efecto, conduzcan a un modelo "energéticamente eficiente, ecológicamente conservacionista y alimentariamente suficiente", que se oponga al modelo de producción de la "pequeña agricultura" que fortalece el sistema agroindustrial y las concepciones de economía verde. Esto no solo destruye la biodiversidad, sino que también lo hace con las prácticas tradicionales de apropiación de la naturaleza que la han conservado (Toledo \& Barrera, 2008; Toledo, 2005).

Es importante comprender la complementariedad de la relación entre naturaleza y cultura, y el reconocimiento de la inconmensurabilidad de los valores y de las visiones de las mismas para avanzar hacia una idea de sustentabilidad fuerte que actúe como paradigma para la economía campesina, concebida desde un enfoque ecointegrador como punto de partida de las relaciones que se tejen entre sujetos y familias y de ellas con el territorio. Esto destaca su sabiduría, racionalidad, significados, prácticas $\mathrm{y}$ formas de manejo que se han demostrado sensibles y cuidadoras del entorno natural. Sin embargo, el desarrollo convencional, "el esquema de la economía ambiental, ignora esta esencia y prácticamente aborda al sujeto separándolo de sus connotaciones ecológicas y sus relaciones con el mundo natural" (Martínez, 2008).

Se trata de un modelo que permite la interacción ecosistema-cultura en equilibrio. Un rasgo importante de la producción campesina es su grado de autosuficiencia, pues son las familias que se involucran en la producción, aquellas que consumen una gran parte de ésta, y de la misma manera producen casi todos los bienes que necesitan. Este proceso se fundamenta en el trabajo familiar, siendo las fuentes de energía predominantes la fuerza animal más que el uso de combustibles fósiles. Asimismo, la producción combina valores de uso y mercancías; no busca el lucro más allá del necesario para el mantenimiento de la producción y de la vida de la familia; por lo tanto, si bien la agricultura es la principal actividad, se combinan prácticas para mantener el ingreso básico como la recolección, el ganado, artesanías, pesca y otros trabajos intermitentes (Toledo, Alarcón \& Barón, 2002). 
Análisis productivistas aseveran que este tipo de economía representa un obstáculo para el desarrollo si no se integra por completo a los grandes circuitos de capital, pues al tener su cadena de comercialización en lo local, no logra mayor relevancia en la economía nacional. Estos análisis ignoran que, según informes como el de Grain (2014), es la economía campesina la que abastece alimentariamente al planeta y la que a su vez es la más productiva.

Reconocer estas virtudes pasa por registrar su importancia no solo en la producción de alimentos de buena calidad, sino también en la conservación de la biodiversidad, el abastecimiento de alimentos para una población creciente sin deteriorar la base de los recursos naturales y la consolidación de mercados locales y redes de cooperación en zonas rurales (Ploeg, 2010; Santacoloma, 2015). De allí que se le atribuya un papel clave en las formas de desarrollo que no dependen del crecimiento y que está sustentada por sus métodos de trabajo, que reconocen el conocimiento y manejo tradicional de los recursos naturales en estos agroecosistemas.

Para Chayanov (1974), Scalerandi (2010) y Berry (2014), la agricultura campesina, que combina los factores clásicos de producción: tierra, trabajo y capital, se diferencia de otras formas de producir en la ruralidad porque sustentan su producción en el uso eficiente de mano de obra familiar, en la utilización de saberes ancestrales, en la diversificación de cultivos y en el flujo permanente de ingresos e innovaciones tecnológicas. Esto aporta considerablemente a las economías locales, a los entornos ambientales que rodean a estos sistemas de producción y a la distribución del ingreso. Del mismo modo, en estos sistemas productivos se encuentran posiciones emancipadoras frente al modelo de desarrollo dominante, cristalizando alternativas de participación, producción, circulación y comercialización de los productos. Sobre la base de una multifuncionalidad del territorio, se da paso a la protección del campesino que contribuye al cierre de la frontera agrícola, al fortalecimiento de la economía campesina y a relaciones de producción solidarias.

Un elemento importante en el análisis es que la economía campesina ha logrado sobrevivir a las políticas económicas rurales más regresivas en un contexto de concentración de la propiedad y de una precaria presencia del estado. Esto ha fortalecido e impulsado proyectos de monocultivos y de agroindustria para el abastecimiento de los mercados globales y ha conducido a una competencia desigual y nociva para esta economía campesina. Pese a todas estas dificultades y desventajas, la economía campesina sigue garantizando la autonomía alimentaria en las regiones más apartadas y, al mismo tiempo, ha permitido un abastecimiento del mercado interno. Al respecto, Eduardo Musso (1998) considera que:

La unidad doméstica campesina organiza sus procesos tomando como punto de referencia y base de cálculo la utilización potencial de su recurso laboral, condicionando a ésta la utilización de los demás en función de los resultados de su mayor recurso disponible. En la práctica, los productores miden los ingresos por el jornal invertido, que es el factor principal, y no por la rentabilidad del capital invertido, que para ellos resulta un factor secundario. (p. 20) 
Comprender esta forma de racionalidad permite aseverar que los campesinos y campesinas no son solamente agricultores. Si bien esta puede ser la actividad preponderante, es siempre complementada por actividades como la pesca, caza, cría de ganado, artesanía o aprovechamiento forestal. Esta combinación de prácticas hace que los elementos ambientales, naturales y transformados se conviertan en un complejo paisaje en el que se entrelazan cultivos agrícolas, bosques primarios y secundarios, jardines domésticos, pastos y corrientes de agua. Esto último demuestra la cercana relación entre la economía campesina y una visión integral del territorio en la que el trabajo humano juega con una estrategia multiuso que se evidencia en el paisaje, en la que se hacen presentes conocimientos propios y heredados que garantizan la subsistencia a través de la manipulación de los componentes geográficos y ecológicos (especies, suelos, topografía, clima, agua y espacio) y de sus procesos (sucesión, ciclos de vida y movimiento de materias) (Toledo, 1993).

Lo anterior permite afirmar que la autosuficiencia campesina, ampliamente basada en una simbiosis permanente con los recursos locales naturales, constituye el punto de partida para un desarrollo alternativo ecológicamente relevante en el que sus prácticas aportan a la sostenibilidad. Lo anterior desestima la imposición de una producción especializada e intensiva, que es viable sólo en tanto haya una autosuficiencia garantizada. Por lo tanto, la subsistencia local y regional, y no la producción comercial dirigida a la exportación, debería ser el primer objetivo productivo de cualquier política de desarrollo rural.

Otro de los argumentos es que en la economía campesina el uso pleno de la mano de obra redunda más en una situación de máxima eficiencia que en el marco de relaciones de solidaridad. En el caso de presentarse situaciones de déficit de mano de obra, esto puede ser solventado con la colaboración de los vecinos o a través de la compra de jornales. Esto lleva a reconocer que hay unos factores monetarios que se interrelacionan con unos no monetarios (Forero, 2010), y es en la relación de ambos que se configura una forma especial de producción de alimentos, valores sociales y relaciones con el territorio

Se podría concluir que la economía campesina es una forma de producción rural agraria en la que la base natural es un medio básico e irremplazable. Este proceso siempre implica la combinación de valores de uso y de cambio y es el resultado de procesos naturales y de fuerzas de mercado que actúan sobre el campesino como productor y consumidor. En el contexto de conflicto armado, esta forma de producción rural agraria estuvo condicionada por dos cadenas de circunstancias paralelas descritas por Rodríguez, Rodríguez y Durán (2017, pp. 23-25). La primera caracterizada por:

i) Los recursos naturales fueron fuente de financiamiento de los grupos armados y aportaron al mantenimiento de este.

ii) Al ubicarse en lugares de difícil acceso, sus territorios sirvieron como refugios para los actores armados. 
iii) El acaparamiento de tierras fue utilizado como estrategia de contención, protección y control por parte de los actores armados que permitió alianzas con empresarios privados y facilitó el desarrollo de proyectos con altos impactos ambientales.

iv) Las zonas boscosas fueron escenarios para otros medios de financiamiento como el secuestro y los cultivos de uso ilícito.

Y la segunda (pp. 35 -37):

i) La presencia de actores armados blindó estos territorios biodiversos del acceso de otras poblaciones o de proyectos de desarrollo que sirvió para conservar estas zonas para sacarlas del mapa para la explotación a gran escala y para recuperar áreas que antes estaban expuestas a diferentes tensiones.

ii) Los actores armados creaban zonas "fuera de límites" en donde ni el Estado ni las empresas pudieron entrar.

iii) La dinámica de conflicto llevó a la creación de normas y reglamentos que conciliaron la actividad productiva y de protección ambiental.

Estas redes causales que se dieron en paralelo se han venido transformando con el desescalamiento del conflicto armado interno y con las nuevas dinámicas espaciales incentivadas por la implementación del acuerdo de paz, que tiene su expresión en la instalación de nuevos asentamientos rurales denominados en un inicio como "Zonas Veredales de Transición y Normalización (ZVTN)" y ahora "Espacios Territoriales de Capacitación y Reincorporación (ETCR)". En estos se concentraron en un inicio las personas de la guerrilla de las FARC-EP, que en cumplimiento de lo acordado harían su desarme y que ahora con sus familias llevan a cabo el proceso de reincorporación, en la mayoría de los casos colectiva y comunitaria. Vale aclarar que, por los incumplimientos del gobierno nacional y la falta de garantías para su subsistencia, muchos de ellos han decidido continuar su reincorporación fuera de estos espacios, pero manteniendo su vida colectiva en lo que se denominan Nuevas Áreas de Reincorporación.

En general, estos asentamientos rurales se encuentran al interior de áreas que el Acuerdo de Paz definió como subregiones para los Planes de Desarrollo con Enfoque Territorial (PDET), las cuales tienen en común haber sido afectadas en mayor medida por el conflicto, presentar mayores índices de pobreza y tener presencia de economías ilegales. Unas 15 de las 16 subregiones se encuentran en áreas de especial interés ambiental y cuentan con áreas protegidas en su interior. 


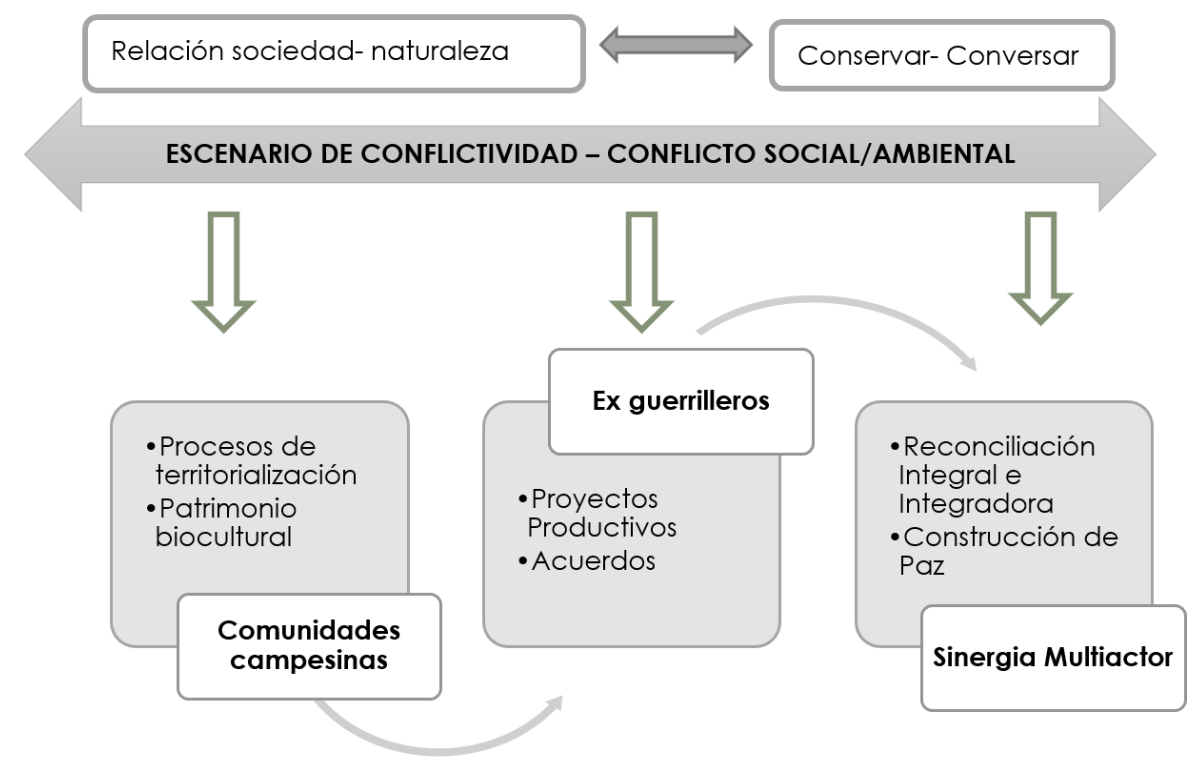

Figura 6. Multifuncionalidad de la vida campesina y excombatiente. Fuente: Elaboración propia.

Es así como el proceso de reincorporación que se está llevando a cabo no solo es un tránsito a la vida civil, sino que comprende la configuración de nuevos relacionamientos con las comunidades campesinas y en conjunto con el ecosistema. Ante esta situación, se llevan a cabo proyectos de cooperación internacional que han definido como líneas de acción incentivar procesos de:

i) Monitoreo de bosques comunitario en donde más allá de obtener datos o levantar información, lo que busca es generar acciones participativas y comunitarias que prevengan conflictos a partir de una estrategia que comprende 6 líneas de acción: bosque, agua, biodiversidad (fauna y flora), patrimonio cultural, y de manera transversal, preservación y cuidado del bosque (incluye restauración-reforestación) y conservación y uso sustentable. Lo anterior con el objetivo de generar condiciones de sustentabilidad ecosistémica y alimentaria.

ii) Cuantitativamente se tiene la meta de restaurar aproximadamente 140 hectáreas en los municipios de Mesetas, Uribe y la Macarena en el Meta y San Vicente del Caguán en el Caquetá.

iii) Para avanzar en este objetivo de restauración en los ETCR, se ubicaron viveros de especies nativas y lugares para la producción de abono propio.

iv) Reconociendo que durante el conflicto ya había una relación entre FARC y las comunidades campesinas, también se busca oficializar agendas ambientales, las cuales pretenden ser respuesta a los conflictos socioambientales de la región a partir de actividades comunitarias definidas en conjunto. 
v) Estas agendas incorporan normas ambientales que hacían parte de manuales de convivencia durante el conflicto. En ellas se incluía la prohibición de caza, la pesca con red, la comercialización de algunas maderas y especies vegetales, la conformación de potreros comunitarios para ganado, cañeros rotativos (potrero para restauración pasiva, también llamado "enrastroje" cañero), porcentajes de uso en las fincas (60 \% en producción y $40 \%$ en "montaña”), apoyo en la construcción de pozo séptico en las fincas y prohibición de cocheras cerca de la ronda de los ríos.

Estas normas ambientales buscan ser retomadas de manera dialogada y colectiva. Luego de la firma del acuerdo y la concentración de la guerrilla en las ZVTN se dejaron de cumplir, lo cual se solapó con la ausencia del gobierno y sus instituciones en estos territorios, donde no se conocía una autoridad diferente a la insurgente y la autoridad ambiental, por la visión de conservación antes descrita, resulta totalmente ilegítima.

Lamentablemente, el gobierno nacional ha decidido continuar y profundizar esta forma de relacionamiento con las comunidades campesinas, lo cual ha resultado en la criminalización, persecución y judicialización de familias asentadas al interior del Sistema de Parques Nacionales Naturales. Lo anterior lleva a concluir que el proceso de implementación del acuerdo de paz debe tener en cuenta las relaciones ambientales en estas zonas altamente biodiversas, comprender y fortalecer las dinámicas comunitarias de conservación e integrar de manera efectiva la institucionalidad estatal a estos territorios.

\section{REFERENCIAS}

Beltrán, Y. (2016). La biocolonialidad en las relaciones entre investigadores de la biodiversidad y las comunidades en Colombia. Revista Tabula Rasa, 24, 213-240.

Berkes, F. y Davidson-Hunt, I. (2006). Biodiversity, Traditional Management Systems, and Cultural Landscapes: Examples from the Boreal Forest of Canada. Int Soc Sci J,58(187), 35-47.

Berry, A. (2014, marzo). La economía campesina. En Debates sobre la problemática agraria. Conferencia dictada en el marco de la cátedra Manuel Ancízar, Universidad Nacional de Colombia. Recuperado de http://www.unradio.unal. edu.co/nc/categoria/cat/catedra-manuel-ancizar/pag/1.html

Boyce, J. (2013). Economics, the environment and our common wealth (1 Ed.). Cheltenham: Edward Elgar Publishing Limited.

Chayanov, A. (1974). La organización de la unidad doméstica campesina. Buenos Aires: Ediciones Nueva Visión.

Davidson-Hunt, I., Turner, K., Te Pareake Mead, A., Cabrera- Lopez, J., Bolton, R., Idrobo, C.J. (2012). Biocultural Design: A New Conceptual Framework for Sustainable Development in Rural Indigenous and Local Communities. SAPIENS, 5(2), 33-45.

Denevan W. (1992). The Pristine Myth: the Landscape of the Americas in 1492. Ann Assoc Am Geogr., 82(3), 369- 385. 
Forero, J. (2010). El campesino colombiano. Entre el protagonismo económico y el desconocimiento de la sociedad. Pontificia Universidad Javeriana. Facultad de Estudios Ambientales y Rurales.

Gómez-Pompa, A. y Kaus, A. (1992). Taming the Wilderness Myth. BioScience, 42(4), 271-279.

Grain. (2014). Hambrientos de Tierra. Recuperado de https://www.grain.org/es/article/entries/4956-hambrientos-de-tierra-los-pueblos-indigenas-y-campesinos-alimentan-al-mundo-con-menos-de-un-cuarto-de-la-tierra-agricola-mundial\#sdfootnote37sym.

Gudynas, E. (2011). Los derechos de la naturaleza en serio. Respuestas y aportes desde la ecología política. En Acosta A. y Martínez E (Comps.), La naturaleza con derechos. De la filosofía a la política (pp. 239-286). Abya Yala, Quito.

Hanson, T., Brooks, T., Da Fonseca, G., Hoffman, M., Lamoreux, J., Machlis, G... (2009). Warfare in Biodiversity Hotspots. Conservation Biology, 23(3), 578-587.

Leopold, A. (2003). Estética de la conservación. Revista Mientras Tanto, 87, 121-131.

Maffi, L. y Woodley, E. (2010). Biocultural Diversity Conservation: A Global Sourcebook. London: Earthscan.

Martínez, A. (2004). El ecologismo de los pobres. Conflictos ambientales y lenguajes de valoración (Primera edición). Barcelona: Icaria AntrazytFlacso.

Martínez. A. (2008). Conflictos ecológicos y justicia ambiental. Revista Papeles, 103. Recuperado de goo.gl/7787iX

Maya, A. (2013). El reto de la vida. Ecosistema y cultura, una introducción al estudio del medio ambiente (Segunda Edición). Bogotá, D. C.: Ecofondo.

McNeely, J. (1994). Áreas protegidas para el siglo XXI: Trabajando para proporcionar beneficios a la sociedad. Recuperado de http://www.fao.org/docrep/v2900s/v2900s03.htm

Mcshane, T.O., Hirsch, P.D., Tran, C.T., Songorwa, A.N., Kinzig, A., Monteferri, B. (2011). Hard Choices: Making Trade-offs Between Biodiversity Conservation and Human Well-being. Biol Conserv, 144(3), 966-972.

Musso, E. (1998). Productores y sus organizaciones en zonas cocaleras del Perú. Un enfoque para la investigación agraria hacia el desarrollo alternativo. Lima: IICA-GTZ.

Nemogá, G. (2016). Diversidad biocultural: innovando en investigación para la conservación. Acta biol. Colomb, 21(1). DOI: 10.15446/abc.v21n1sup.50920.

Organización de las Naciones Unidas para la Alimentación y la Agricultura (FAO). (2004). De la biodiversidad al servicio de la seguridad alimentaria. Sala de prensa. Recuperado de http://www.fao. org/Newsroom/es/news/2004/42621/index.html

Oviedo, G., Maffi, L. y Larsen, P. (2000). Indigenous and traditional peoples of the world and ecoregion conservation: an integrated approach to conserving the world's biological and cultural diversity, with accompanying map. Gland, Suiza: WWF International. 
Persic, A. y Martin, G. (Eds.). (2008). Links between biological and cultural diversity-concepts, methods and experiences. Report of an International Workshop. París: UNESCO.

Ploeg, J. (2010). Nuevos campesinos. Campesinos e imperios alimentarios. Perspectivas agroecológicas. Barcelona: Icaria Editorial.

Posey D. (1985). Indigenous management of tropical forest ecosystems: the case of the Kayapó Indians of the Brazilian Amazon. Agroforestry Systems, 3-2,39- 158. Doi: 10.1007/BF00122640

Posey D. (1997). The Kayapó: the Role of Intellectual Property in Resource Management in the Brazilian Amazon. En Posey, D.A. y Dutfield, G. (Editors), Indigenous Peoples and Sustainability: Cases and Actions (pp. 240-254). Utrecht: IUCN and International Books.

Rodríguez, C., Rodríguez, D. y Durán, H., (2017). La Paz Ambiental, Retos y propuestas para el Posacuerdo. Bogotá: Dejusticia.

Safford, F, y Palacios, M. (2012). Historia de Colombia: País Fragmentado, Sociedad Dividida. Recuperado de http://babel.banrepcultural.org/cdm/ref/collection/p17054coll10/id/2581

Santacoloma-Varón, L. (2015). Importancia de la economía campesina en los contextos contemporáneos: una mirada al caso colombiano. Entramado, 11(2), 38-50.

Scalerandi, V. (2010). El lugar del campesino en la sociedad: aportes del marxismo a la comprensión de la articulación entre campesinos y modos capitalistas de producción. Revista de Antropología Ciencias Sociales Kula, (2).

Serje, M. (2005). El revés de la Nación. Territorios salvajes, fronteras y tierras de nadie. Bogotá: Universidad de los Andes.

Toledo, V. (1993). La Racionalidad Ecológica de la Producción Campesina.En Sevilla Eduardo y González de Molina Manuel (Eds.), Ecología, campesinado e historia (197-218).

Toledo, V. (2005). Repensar la conservación: ¿áreas naturales protegidas o estrategia bioregional? Gaceta ecológica, 77, 67-83.

Toledo, V. y Barrera-Bassols, N. (2008). La memoria biocultural. La importancia ecológica de las sabidurías tradicionales. Barcelona: Icaria Editorial.

Toledo, V., Alarcón, C. y Barón, L. (2002). Revisualizar lo rural: un enfoque socioecológico. Gaceta Ecológica, 62, 7-20.

Turner, N., Boelscher, M. y Ignace, R. (2000). Traditional ecological knowledge and wisdom of aboriginal peoples in British Columbia. Ecol Appl., 10(5), 1275-1287

Uribe, S. (2013). Historical ethnography of a road in the Putumayo region of Colombia. The London School of Economics and Political Science (LSE).

Wolff, F. (2004). Industrial Transformation and Agriculture: Agrobiodiversity Loss as Sustainability Problem. En Klaus Jacob, Manfred Binder y Anna Wieczorek (Eds.). Governance for Industrial Transformation. Proceedings of the 2003 Berlin Conference on the Human Dimensions of Global Environmental Change. 